\title{
La représentation des métiers chez des adolescent(es) scolarisé(es) au collège et au lycée : « Du mouvement mais pas de changement »
} Titre d'un rapport d'A. Thibaud (1995) remis au ministre de l'Education du Québec, coordination féminine Occupational Representations among Teenagers at School and Grammar School

Nicole Mosconi et Biljina Stevanovic

\section{(2) OpenEdition}

\section{Journals}

Édition électronique

URL : http://journals.openedition.org/travailemploi/3766

DOI : $10.4000 /$ travailemploi.3766

ISSN : $1775-416 \mathrm{X}$

Éditeur

DARES - Ministère du Travail

Édition imprimée

Date de publication : 15 mars 2007

Pagination : $59-80$

ISBN : ISSN : 0224-4365

ISSN : 0224-4365

\section{Référence électronique}

Nicole Mosconi et Biljina Stevanovic, "La représentation des métiers chez des adolescent(es)

scolarisé(es) au collège et au lycée : "Du mouvement mais pas de changement » », Travail et Emploi [En ligne], 109 | janvier-mars 2007, mis en ligne le 15 mars 2009, consulté le 10 décembre 2020. URL http://journals.openedition.org/travailemploi/3766 ; DOI : https://doi.org/10.4000/travailemploi.3766 


\title{
La représentation des métiers chez les adolescent(es) scolarisé(es) au collège et au lycée
}

\author{
«Du mouvement mais pas de changement» $\left(^{*}\right)$ \\ Biljana STEVANOVIC $(* *)$ \\ Nicole MOSCONI $(* * *)$
}

C'est pendant l'adolescence, à un moment de grands remaniements de la personnalité, que filles et garçons doivent décider de leurs orientations et projets professionnels. Malgré les actions mises en place pour lutter contre les stéréotypes sexués et malgré l'évolution de la place des femmes sur le marché du travail, les représentations des adolescent(es) n'ont que très peu évolué, reproduisant la division sexuée du marché du travail. Comment les représentations se construisent-elles tout au cours de la scolarité? Quels sont les facteurs qui influencent les représentations des filles et des garçons et les poussent à s'engager dans des filières puis des carrières professionnelles différenciées? Cet article propose un recensement de la littérature francophone et anglophone publiée entre 1984 à 2004 sur le sujet, regroupée autour des quatre grands courants théoriques qui s'imposent dans l'étude des facteurs psychosociologiques à l'origine des choix professionnels: le modèle de Gottfredson et la carte cognitive de représentations des professions; le modèle de l'orientation de genre de Sandra Bem; le modèle de Holland et du «choix vocationnel »; le modèle de l'apprentissage social de Bandura, dont le concept-clé de sentiment de compétence ou d'efficacité personnelle paraît avoir une pertinence particulière pour expliquer le développement de la vie professionnelle des femmes.

La différenciation selon le genre détermine fortement, dans la plupart des pays occidentaux, les caractéristiques du marché de l'emploi. En matière de parcours scolaire, d'insertion professionnelle, de statut socio-professionnel, le genre reste en effet, avec l'origine sociale, un facteur essentiel d'explication des inégalités. L'orientation scolaire différenciée des filles et des garçons apparaît à cet égard comme déterminante. Bien que les filles réussissent en moyenne mieux leur scolarité que les garçons, qu'elles redoublent moins qu'eux et obtiennent plus souvent leurs diplômes avec mention, au moment des grands paliers d'orientation, elles se dirigent vers des voies moins rentables en termes professionnels et «perdent ainsi une partie du bénéfice de cette meilleure réussite scolaire» (COUPPIÉ, EPIPHANE, 2001).

La sous-représentation des filles dans les filières scientifiques et technologiques et dans les métiers scientifiques et techniques industriels est un fait avéré par toutes les recherches sur cette question.

(*) Titre d'un rapport d'A. Thibault (1995) remis au ministre de l'Éducation du Québec, coordination féminine.

(**) Docteure en sciences de l'éducation, ATER en sciences de l'éducation à l'université de Basse Normandie.

(***) Professeure en sciences de l'éducation à l'université Paris X Nanterre.
Le métier d'ingénieur en France en est un exemple remarquable: en 2002, les 80000 femmes ingénieurs qui exercent une activité professionnelle ne représentent que $16 \%$ de l'ensemble des ingénieurs (1). Ce fait s'accompagne, depuis une dizaine d'années, d'une baisse importante du nombre d'étudiants masculins dans les filières scientifiques universitaires, baisse que des programmes tentent de palier en augmentant le nombre des étudiantes. Plus généralement, les efforts faits pour améliorer l'orientation scolaire et professionnelle des filles n'ont pas eu les résultats escomptés (2). Tant dans les pays anglophones que francophones, la différenciation sexuée des orientations perdure. Les filles s'orientent vers les sections littéraires et tertiaires,

(1) Données 2003 de l'Association française des femmes ingénieurs.

(2) En 1985, un programme d'action, «Égalité des chances entre filles et garçons à l'école», financé par la CEE, avait pour objectif de faire prendre conscience aux élèves des stéréotypes associés aux métiers supposés «féminins» ou «masculins». En France, après la loi d'orientation du 10 juillet 1989 stipulant que «les lycées et les établissements d'enseignement supérieur sont chargés [...] de favoriser l'égalité entre les hommes et les femmes», une "convention pour la promotion de l'égalité des chances entre les filles et les garçons, les hommes et les femmes dans le système éducatif» a été signée en 2000 par plusieurs ministres. 
les garçons, vers les sections scientifiques et techniques industrielles. Ces orientations entraînent inévitablement une division sexuée du marché de l'emploi. Elles préfigurent certaines des hiérarchies les plus classiques entre les sexes (médecin - infirmière, homme cadre - femme assistante ou secrétaire, homme professeur d'université - femme professeur de collège ou de lycée, homme contremaître - femme OS...) qui signent la subordination féminine dans la sphère professionnelle comme dans la sphère familiale.

Pour autant, des changements se dessinent: au cours des vingt dernières années, leur taux de scolarisation s'est accrue, et les femmes ont opéré des avancées au sein des professions libérales, des professions administratives et commerciales ou parmi les cadres. La conséquence en est une évolution de l'emploi des femmes vers une bipolarisation: les plus diplômées ont des opportunités d'emploi plus nombreuses et plus variées, permettant même d'intégrer la dimension familiale dans les choix réalisés; à l'autre bout de l'échelle, la situation s'est aggravée pour l'insertion des femmes faiblement diplômées et non diplômées, davantage que pour les hommes. Elles sont beaucoup plus fréquemment au chômage et se retrouvent beaucoup plus souvent que les hommes dans des emplois faiblement rémunérés, précaires ou à temps partiel.

Les recherches sur l'orientation scolaire ont conduit à interroger la place des représentations des métiers chez les filles et les garçons au collège et en lycée. Pour comprendre cette ségrégation des emplois et sa résistance au changement malgré la mixité des systèmes scolaires, les chercheurs se sont efforcés depuis plusieurs années de les interpréter en faisant l'hypothèse qu'elles dépendaient des représentations qu'adolescentes et adolescents scolarisé(es) au collège et au lycée se font des métiers, à l'heure des projets professionnels.

C'est pendant l'adolescence, à un moment de grands remaniements de la personnalité, que doivent se décider les orientations et les projets professionnels. Étudier les représentations des métiers chez les élèves des deux sexes aux moments cruciaux de l'orientation scolaire et leurs liens avec les stéréotypes de sexe encore fortement prégnants dans nos sociétés devrait permettre de comprendre comment se font ces choix différenciés. Comment les représentations se construisent-elles tout au cours de la scolarité? Quels sont les facteurs qui influencent les représentations des filles et des garçons et les poussent à s'engager dans des filières puis des carrières professionnelles différenciées?

Telles sont les questions qui ont guidé l'analyse de la production scientifique sur les orientations sexuées des jeunes que nous proposons ici. Nous avons limité notre investigation à la littérature francophone et anglophone publiée entre 1984 à 2004 ayant pour objet les déterminants de l'orien-
Encadré 1

\section{Méthodologie de l'étude}

Ce survey de littérature a été élaboré dans le cadre d'une convention entre la direction de l'animation de la recherche, des études et des statistiques du ministère de l'Emploi, de la Cohésion sociale et du Logement (DARES) et l'université de Paris X-Nanterre.

Le corpus d'étude est composé de soixantehuit études et recherches, dont trente-huit francophones et trente Anglo-Saxonnes. Notre méthode de travail a consisté à chercher des travaux portant sur la représentation des métiers dans des revues de sociologie, de psychologie, et d'études féministes qui traitent des questions d'éducation et d'orientation scolaire et professionnelle. Une préoccupation souvent mise en avant dans la littérature anglophone est le développement de la carrière des adolescentes et les éléments qui contribuent au développement de la carrière scientifique et technique. Ces études apportaient de nouvelles perspectives sur la thématique des choix professionnels. En prenant en compte ces nouveaux éléments, notre objet d'étude a porté sur les représentations des métiers des adolescents et adolescentes et sur le développement de leur choix professionnel. La tranche d'âge concernée se situait de 11 ans à 19 ans.

\section{Revues francophones exploitées}

Orientation Scolaire et Professionnelle, Questions d'orientation, Les cahiers internationaux de psychologie sociale, L'Indécis, Bulletin de psychologie, Éducation permanente, Recherches féministes, Carriérologie, Orientation et Formation Professionnelle, Revue de psychologie d'éducation et d'orientation.

\section{Revues anglophones}

Journal of Vocational Behavior, Sex Roles: A Journal of Research, Journal of Adolescent Research, The School Counselor, Career Development Quarterly, Journal of Counseling Psychology, Rœper Review, British Journal of Guidance and Counseling, The Sociological Quarterly, Adolescence, British Journal of Educational Psychology.

Les soixante-huit documents retenus ont été classés selon les thèmes suivants: représentations des métiers «masculins", "féminins» et «mixtes», représentations des métiers selon le niveau scolaire, représentations des métiers nontraditionnels et des métiers scientifiques, cadre mixte et non-mixte de l'enseignement et représentations des métiers, aspirations, sentiment de compétence et le développement de carrière, programmes de sensibilisation des filles aux métiers non traditionnels, influence familiale sur la représentation des métiers.

tation chez les adolescent(es), et leur influence sur 
leurs représentations des métiers. Il ressort que les travaux sur ces questions s'organisent autour de quatre courants théoriques qui dominent à la fois la littérature anglaise, américaine et française: le modèle de Gottfredson, qui propose le concept d'une carte cognitive de représentations des professions; le modèle de l'orientation de genre de Sandra Bem; le modèle de Holland et sa théorie du « choix vocationnel»; le modèle de l'apprentissage social de Bandura, avec son concept-clé du sentiment de compétence ou d'efficacité personnelle (selfefficacy), qui semble exercer une forte influence sur les recherches récentes de ce champ.

\section{Le modèle de Gottfredson}

Linda GotTFREDSON (1981) a proposé une modélisation des représentations des professions en les classant selon deux axes: masculinité/féminité et niveau de prestige. Elle montre que tous les enfants prennent conscience en premier lieu de la différence sexuée des emplois, puis que les différentes fonctions ont des niveaux de prestige social inégaux. À partir de treize ou quatorze ans, tous les adolescents disposent ainsi d'une carte cognitive unique pour se représenter les professions. Parmi les champs possibles de travail, une zone des choix de carrière acceptables peut être tracée selon trois critères: la compatibilité du sexe perçu de chaque métier avec l'identité de genre, la compatibilité du niveau perçu de prestige de chaque métier avec le sentiment d'avoir les capacités pour accomplir ce travail, et la volonté de faire le nécessaire pour obtenir le travail désiré. Cette carte des carrières acceptables déterminera ultérieurement les changements de métiers possibles.

La situation actuelle du marché de l'emploi donne une image quasi identique pour tous les pays du monde, celle d'une connotation féminine pour les métiers du secteur tertiaire, où l'insertion est plus difficile et les salaires moins élevés, celle d'une concentration des hommes dans les secteurs techniques et industriels, où les possibilités d'insertion et de revenus sont beaucoup plus favorables. De nombreux travaux francophones et anglophones s'inspirent de la théorie de Linda Gottfredson: l'étude de la représentation des métiers selon ces deux dimensions montre clairement les différences sexuées des représentations : les filles marquent leurs préférences pour les métiers de soin ou du social, alors que les garçons préfèrent les métiers scientifiques et techniques ayant comme caractéristique principale un salaire élevé. HutEau et MARRo (1986), dans leur étude sur la représentation du mot «travail», constatent que les préoccupations des filles sont liées à la vie sociale et à la conciliation des rôles familiaux et professionnels, et que celles des garçons sont liées au salaire. StockARD et MCQEe (1990), réalisent une enquête par entretien auprès de 486 adolescents scolarisés en classe de quatrième dans l'état de l'Orégon, pour étudier leurs préférences pour vingt et un métiers différents. Ils leur ont proposé trois types de modèles professionnels à l'aide d'images: le premier type mettait au travail une personne de même sexe que l'enfant; le deuxième type mélangeait les sexes au travail; le troisième type développait les images en mettant au travail un homme et une femme, mais en renversant les rôles, quand il s'agissait des métiers liés typiquement à l'un ou à l'autre sexe. La consigne donnée était la suivante: «Ces cartes montrent des personnes faisant différents métiers. Je veux que vous regardiez chaque image et pensiez combien vous voudriez ou détesteriez exercer ce travail quand vous serez grand». Les résultats indiquent que le facteur le plus influent sur les préférences professionnelles des enfants est leur sexe. Les métiers préférés des garçons sont les métiers scientifiques, ou les métiers d'officier de police, artiste, fermier, charpentier et architecte. Les métiers préférés des filles sont: institutrice, professeur de lycée, fermière, artiste, secrétaire et épicière. Une plus grande conscience des caractéristiques professionnelles n'élimine pas les différences de sexe dans les préférences professionnelles. Lors de l'entretien, les images présentées aux élèves ayant renversé les modèles stéréotypés des métiers n'ont pas réussi à changer leur perception stéréotypée du monde du travail. Leurs perceptions des caractéristiques professionnelles ont affecté leurs préférences indépendamment du sexe de la personne représentée sur l'image. Si les garçons croient que les chauffeurs de camion, les pompiers, les scientifiques, et les réparateurs de TV gagnent plus d'argent et exercent un métier plus prestigieux que les pilotes, ils vont préférer ces métiers. En revanche, même si les filles croient que le métier de pilote est plus prestigieux ou qu'il offre un salaire élevé, ces croyances n'augmentent pas leur préférence pour le métier qu'elles considèrent comme masculin. Les études de Guichard $(1992,1994)$ et de WACH $(1992)$ montrent que le métier d'ingénieur est considéré comme un métier d'homme, les métiers de secrétaire et assistante sociale comme des métiers de femme. Seul le métier de médecin est considéré comme convenant aux deux sexes.

Dans une étude sur l'imaginaire professionnel menée auprès de 225 adolescents en classe de $6^{\mathrm{e}}$ (119 filles et 106 garçons) scolarisés dans trois collèges de Bordeaux, Bernadette Dumora (2002) s'est proposée de tester trois hypothèses: 1'hypothèse de la stéréotypie des préférences professionnelles, l'hypothèse de la différence de ces préférences en fonction du sexe et l'hypothèse d'une différence de structure des préférences entre garçons et filles. L'auteure a demandé aux adolescents de répondre en temps libre à la question: "Quelle(s) profession(s) aimerais-tu exercer plus tard? ». Cette étude montrait que les préférences exprimées par les 
filles et les garçons ont en commun de se référer aux professions en évoquant des images concrètes: les gestes professionnels (soigner, enseigner, poser pour les photographes... ou photographier, conduire des engins, transporter, attraper et punir...) ou les «objets» sur lesquels portent les professions (le bébé, l'élève, le malade, l'animal, le malfaiteur, les véhicules...) qui sont le support privilégié. Selon l'auteure, la force des stéréotypes est évidente et peut s'expliquer par l'adhésion des adolescents à certaines images et valeurs de la société que les discours et les médias diffusent.

L'auteure fait ensuite l'hypothèse de l'existence d'une structure, d'une organisation ordonnée des préférences, différente d'une répartition aléatoire. Les résultats signalent l'existence de chaînes significatives chez les filles et de l'absence de chaînes significatives comparables chez les garçons. Chez les filles, la chaîne puéricultrice-institutrice-professeur est «développementale» et assure quasi chronologiquement le passage entre le pôle soin, la puéricultrice intervenant auprès du nourrisson, et le pôle enseignement, l'institutrice intervenant auprès de l'enfant et le professeur auprès de l'adolescent. Chez des garçons, la chaîne la plus centrale est celle qui relie les deux professions les plus fréquentes, pilote à sportif, mais leur corrélation est moins forte que celle de l'enfance chez les filles.

L'hypothèse de la préférence professionnelle en fonction de sexe, et ceci pour la majorité des professions présentes, se vérifie, car les réponses des garçons et des filles diffèrent et vont dans le sens de la masculinité/féminité de la carte cognitive de Gottfredson.

Dans le processus du développement du choix professionnel, les individus font des compromis. Le besoin de faire des compromis peut être attribué aux différences entre les aspirations professionnelles d'un individu et les métiers qui lui sont accessibles. Les adolescents scolarisés en $6^{\mathrm{e}}$ expriment bien un imaginaire professionnel, mais un imaginaire stéréotypé, sexué et peu confronté aux exigences de la réalité. Quand l'adolescent(e) grandit, le choix des différents métiers devient plus réaliste et complexe, et le compromis peut devenir nécessaire si des aspirations sont perçues comme peu réalistes ou inaccessibles. Selon la théorie du compromis de GotTfredson $(1981,1996)$, le compromis est un processus par lequel les individus changent leurs aspirations pour des choix professionnels plus réalistes et plus accessibles. Deux types de compromis sont identifiés par Gottfredson: anticipé et empirique. Des compromis anticipés se produisent quand un individu s'aperçoit que le métier qu'il a le plus désiré n'est pas un choix accessible ou réaliste. Les compromis empiriques se produisent quand un individu modifie ses aspirations en réponse aux expériences qu'il a faites en essayant d'obtenir un emploi. Puisque les compromis anticipés sont des réponses aux perceptions de l'inaccessibilité, sans expériences réelles sur le marché du travail, ils peuvent se produire plus tôt dans le processus du développement du choix professionnel.

Amstrong et Crombie (2000) ont mené une enquête longitudinale et étudié les stratégies de compromis dans les aspirations professionnelles auprès des 502 adolescent(es) (245 garçons et 257 filles) issu(es) de trois écoles d'une ville canadienne. Leurs aspirations et leurs espérances professionnelles ont été évaluées au moyen d'un questionnaire distribué pendant trois ans, lorsqu'ils étaient scolarisés en $8^{\mathrm{e}}, 9^{\mathrm{e}}$ et $10^{\mathrm{e}}$ année. Les résultats montrent que 45 à $48 \%$ d'adolescent(es) changent leurs aspirations professionnelles à n'importe quel niveau de la scolarité, alors que $77 \%$ manifestent un changement de choix professionnel au moins une fois en trois ans. L'analyse des différences entre leurs choix idéaliste et réaliste montre que le changement des aspirations des filles porte sur des métiers traditionnellement féminins, alors que celui des garçons vise les métiers masculins. Les filles ont des aspirations élevées portant majoritairement sur les métiers traditionnellement féminins.

Ces recherches montrent que la représentation des métiers selon leur statut de masculinité/féminité, ou de prestige, a peu évolué au cours des vingt dernières années et ceci pour nos deux champs d'étude. Les filles continuent à manifester leurs préférences pour les métiers traditionnellement féminins, pour des métiers de soin ou du social, alors que les garçons préfèrent les métiers scientifiques et techniques, ayant comme caractéristique principale un salaire élevé. Les choix des métiers qui illustrent le mieux ces modèles de rôles stéréotypés sont: celui d'ingénieur considéré comme un métier masculin et ceux de secrétaire, d'infirmière et d'assistante sociale considérés comme des métiers féminins. D'après ces études et conformément à la théorie de Gottfredson, il apparaît que les filles ont des représentations des professions stéréotypées en adéquation avec les rôles sociaux traditionnellement assignés aux femmes et qu'elles jugent plus favorablement des professions considérées comme «typiquement féminines». Les résultats obtenus sur les changements d'aspirations confirment également la théorie du compromis de Gottfredson.

\section{Le modèle des orientations de genre de Sandra Bem}

Les travaux de Sandra Bem sur l'orientation de genre ont également influencé de nombreuses recherches sur ce thème. Sandra Bem refuse l'idée que les catégories masculin-féminin doivent être considérées comme des catégories binaires qui classeraient nécessairement les individus dans l'une ou l'autre catégorie et qu'il doive y avoir correspon- 
dance stricte entre le sexe d'état civil des individus et leur féminité ou masculinité, de sorte que ceux pour qui cette correspondance ne s'observe pas seraient considérés comme anormaux. Sandra Bem reprend la distinction des théories féministes entre sexe biologique, sexe d'état civil et genre, entendu comme construction sociale de la masculinitéféminité et des rôles de sexe. Dès lors, elle montre qu'on peut déterminer des degrés de féminité et de masculinité selon les individus et elle construit un test comprenant des items de traits et de comportements caractérisés comme féminins, masculins ou neutres (convenant aussi bien aux femmes qu'aux hommes).

Partant de là, la notion d'orientation de genre lui permet de distinguer quatre groupes d'individus: les individus «féminins» qui ont un haut score de féminité, un faible score de masculinité et un faible score aux item neutres, les individus masculins qui ont un faible score de féminité, un haut score de masculinité et un faible score aux item neutres, les androgynes, qui ont un haut score de féminité et de masculinité et un faible score aux item neutres et les indifférenciés, qui ont un faible score de féminité et de masculinité et un score élevé sur les item neutres. Elle montre aussi que les individus les plus efficients ne sont pas ceux dont l'orientation de genre est féminine ou masculine mais plutôt ceux qui sont androgynes. Ainsi les transgressions des normes de sexe ne sont pas le signe d'un déséquilibre chez les individus mais seraient plutôt le fait de personnalités plus actives et plus créatives.

Une étude par questionnaire de WulfF et STEITZ (1997) auprès de quarante filles âgées de 16 à 18 ans, dont vingt appartenant à une classe préparatoire à l'université (classe supérieure de mathématiques), et vingt autres à une classe professionnelle de cosmétologie, teste leur orientation de genre. L'hypothèse était que les filles qui auraient choisi la cosmétologie, orientation typiquement féminine, manifesteraient une orientation de genre féminine et que les filles qui auraient choisi une orientation nontraditionnelle, plutôt considérée comme masculine, manifesteraient une orientation de genre androgyne. Les résultats infirment ces hypothèses : l'orientation de genre dominante dans la classe de cosmétologie était androgyne, alors que dans la classe de niveau supérieur de mathématiques les filles montraient une orientation de genre féminine. Pour expliquer ce résultat paradoxal, les auteurs, supposent que le développement d'une orientation androgyne a été facilité, chez ces filles qui sont dans la voie professionnelle et qui ont déjà choisi leur métier, par leur confiance en elles et leur estime de soi - traits qui correspondent à une orientation de genre androgyne dans le modèle de Sandra Bem. En revanche, les filles qui sont dans la filière de préparation à l'université, en raison du vaste choix de carrières qui s'offre encore à elles et des décisions qu'elles n'ont pas encore prises à ce sujet, n'ont pas encore pu se forger une confiance en elles, une assurance et une estime de soi, ce qui expliquerait leur score de féminité élevé.

\section{La théorie du «choix vocationnel » de Holland}

Holland (1966) propose une théorie du «choix vocationnel», en distinguant six catégories d'individus en fonction d'intérêts professionnels (réaliste, investigateur, artistique, social, entreprenant, conventionnel), correspondant à des profils de personnalités différents. Pour lui la prédiction du choix vocationnel est l'interaction entre la personnalité d'un individu et de son environnement. Un individu est décrit par les caractéristiques suivantes : héritage, activités, intérêts, compétences, disposition (concept de soi, perception de soi et du monde, valeurs, sensibilités aux influences de l'environnement, traits de personnalité); l'environnement par: maison, école, amis et autres personnes de l'entourage. Holland a représenté cette typologie par un modèle hexagonal définissant les ressemblances psychologiques et les interactions entre les types de personnalité et les environnements. Selon cette théorie, les intérêts professionnels seraient inégalement répartis selon les sexes. Les garçons sont plus intéressés par les carrières situées sur les facteurs «réaliste» (manuels et techniques) et «investigateur» (physicien, chimiste, biologiste, informaticien), alors que les filles sont plus intéressées par les carrières situées sur les facteurs «social» (services sociaux et éducation) et «conventionnel» (secrétariat et comptabilité).

Lors d'une enquête effectuée auprès de 112 collégiens scolarisés en $8^{\mathrm{e}}$ année dans un collège aux Etats-Unis, LAPAN et JINGELESKI (1992) ont pu observer l'impact du genre sur leurs aspirations professionnelles. La variable «jugement de compatibilité entre "moi et travail"» a été examinée sur les six types professionnels. Les effets de genre ont été mis en évidence à travers cette carte. Pour le facteur «entreprenant» dans le secteur de genre-neutre de la carte, aucune différence de sexe n'a été trouvée. Cependant, les garçons ont exprimé des espérances plus élevées que les filles d'atteindre une carrière scientifique, même si ces filles et ces garçons avaient des niveaux de réussite équivalents en mathématiques. Plus les espérances de réussite en mathématiques sont basses, plus les élèves ont la probabilité de s'orienter vers un métier situé sur le facteur «social». Les garçons sont plus intéressés par les carrières situées sur les facteurs «réaliste» et «aventure», et ont une plus grande espérance d'atteindre une carrière située du côté du facteur «investigateur» de «haut-prestige». Les filles sont plus intéressées que les garçons par les carrières situées du côté de facteurs «social» et «conventionnels». Cette étude 
présente des données décrivant les facteurs qui peuvent orienter les collégiens dans le monde du travail. Les conseillers d'orientation peuvent utiliser ces résultats en évaluant les perspectives de carrière des collégiens et les aider à s'orienter.

Nous avons vu que la théorie du «choix vocationnel» de Holland distingue six catégories d'intérêts professionnels correspondant à des profils de personnalités différents. Ces six dimensions ne sont pas indépendantes, mais peuvent donner lieu à une représentation spatiale suivant une structure qui montre les similitudes psychologiques entre les différents types de ce modèle. L'hypothèse structurelle de Holland prévoit une équidistance entre les différents types, définissant ainsi un hexagone équilatéral avec des points équidistants autour de la circonférence. Guglielnie et alii (2004) étudient les intérêts professionnels selon le modèle de Holland et en particulier appliquent les principales procédures de vérification du modèle hexagonal sur un échantillon de lycéens italiens. Un questionnaire portant sur les dénominations des professions, les descriptions d'activités et les types de compétences pour les différentes activités, a été soumis à 534 lycéens. La comparaison des résultats par sexe a montré que les garçons obtiennent un score plus élevé dans le domaine réaliste et conventionnel, tandis que les filles se concentrent davantage sur les domaines artistique et social. Les types investigateur et entreprenant sont par contre équilibrés pour les deux sexes. Selon les auteurs, il apparaît que les filles ont un système de préférences professionnelles moins structuré par rapport aux règles du modèle de Holland. En effet, l'ensemble des intercorrélations entre types pour les filles est moins cohérent $\mathrm{du}$ point de vue du modèle que celui des garçons. Ainsi, dans le contexte italien et dans le cadre des lycées d'un territoire bien délimité, une différence dans la structure des intérêts professionnels se différencie nettement entre les filles et les garçons.

Les derniers travaux considérés prennent en compte un autre facteur, le sentiment de compétence. Une étude récente (TURNER, LAPAN, 2002) menée auprès de 139 élèves scolarisés en $7^{\mathrm{e}}$ et en $8^{\mathrm{e}}$ années dans un collège aux États-Unis, a testé le modèle de Holland avec les hypothèses suivantes: le sexe des élèves, les modèles traditionnels sexués de carrière, le sentiment d'efficacité professionnelle, et la planification et l'exploration de la carrière, en supposant que ces facteurs permettraient de prévoir de manière significative et différentielle les intérêts de carrière des collégiens, à travers les six modèles professionnels de Holland; le soutien parental permettrait de prévoir de manière significative et différentielle le sentiment de compétence professionnelle des adolescents à travers le modèle de Holland; et le soutien parental permettrait de prévoir directement des intérêts de carrière des adolescents à travers le modèle de Holland.
Les résultats montrent que le sentiment de compétence professionnelle et la capacité de planification/exploration professionnelles orientent les intérêts professionnels des jeunes adolescent(es) conformément aux profils du modèle de Hollande, et que les modèles sexués traditionnels de carrière entraînent des intérêts pour les carrières réalistes, investigatrices et sociales. Le facteur de soutien parental n'a pas été directement associé aux intérêts professionnels des jeunes adolescent(es), bien qu'il ait un impact direct sur le sentiment de compétence professionnelle des adolescent(es). Le genre et les modèles de carrières traditionnels ont un effet sur les intérêts professionnels des adolescent(es) dans des carrières réalistes, investigatrices, et sociales, mais pas dans les carrières artistiques, entreprenantes, ou dans les carrières conventionnelles. Les garçons montrent un plus grand intérêt professionnel pour les carrières réalistes et investigatrices, alors que les filles montrent un plus grand intérêt professionnel pour les carrières sociales qu'elles associent à des dispositions féminines. Les auteurs soulignent que le sentiment de compétence professionnelle et la planification/exploration professionnelle sont associés aux intérêts professionnels des adolescentes selon le modèle de Holland. Ils avancent l'hypothèse que la confiance joue un rôle déterminant pour explorer le monde professionnel, trouver l'information sur un métier spécifique, et s'engager dans une planification éducative et professionnelle intentionnelle, peuvent faciliter les aspirations professionnelles des adolescentes pour des carrières qu'elles avaient d'abord perçues comme inaccessibles.

Le modèle de Holland a ouvert des pistes de recherche intéressantes, mais tend aujourd'hui à être supplanté, ou complété, par le modèle de Bandura.

\section{La théorie de l'apprentissage social de Bandura}

Beaucoup d'études sur le développement de la vie professionnelle et les aspirations des adolescents quant au choix de leur futur métier soulignent aujourd'hui l'importance du sentiment de compétence (self-efficacy en anglais).

La notion de «sentiment de compétence» ou d' ' auto-efficacité» s'inscrit dans la théorie sociale cognitive (TSC) de BANDURA (1977, 1982), qui s'oppose au behaviorisme, et avance que ce ne sont pas seulement les environnements qui déterminent les choix individuels, mais que les individus et leurs environnements se déterminent mutuellement. Selon cette théorie, l'apprentissage par observation et l'autorégulation jouent un rôle important dans les représentations. L'être humain est un être intentionnel, capable de se donner des buts et d'en changer. Bandura s'intéresse ainsi aux événements internes et pas seulement aux stimulations externes, 
en particulier au sentiment de compétence. À l'origine, cette notion désignait les croyances de l'individu sur sa propre capacité à se comporter en adéquation au résultat espéré. La définition s'est élargie aux croyances des personnes sur leurs capacités à exercer un contrôle sur leur vie et sur leurs capacités à mobiliser des connaissances et des actions spécifiques pour réaliser une tâche donnée. Le sentiment de compétence influe sur les buts (plus on se sent compétent, plus on peut se fixer des buts difficiles); sur les choix d'activités et sur la persévérance face aux obstacles; sur l'efficacité à résoudre des problèmes; sur l'autorégulation, en orientant l'activité de sélection de situations et d'activités, pour s'engager dans des situations susceptibles d'aboutir à un succès et éviter celles où la personne s'attend à échouer. Bandura suppose ainsi que la perception des chances de réussite peut déterminer en partie le développement de carrière. Le sentiment de compétence facilite des comportements efficaces de planification des carrières.

Hackett et Betz (1981) ont repris cette théorie pour comprendre les choix d'orientation des adolescents et des adolescentes, montrant que ces choix peuvent s'expliquer conjointement par les centres d'intérêt et le sentiment de compétence. On sait que les intérêts scolaires et professionnels sont modelés par les apprentissages et les rôles de sexe et obéissent souvent à des stéréotypes de sexe. En même temps, les deux chercheuses ont souligné l'influence du sentiment de compétence sur ces choix: ainsi, des jeunes ne font pas tel choix d'orientation par manque de compétences objectives, mais parce qu'ils ont un sentiment de compétence faible par rapport à ces études. Ainsi un faible sentiment de compétence en mathématiques, même s'il ne correspond pas à une faiblesse objective, agit comme une barrière vis-à-vis de certaines filières et vis-à-vis d'un grand nombre de métiers qui exigent des "compétences quantitatives». Cette hypothèse de l'influence sociale souligne que le sentiment de compétence peut contribuer à expliquer les différences de choix professionnel liées au sexe. Hackett et Betz ont montré en effet que les filles s'estiment généralement moins compétentes que les garçons pour les professions scientifiques. Pour expliquer ce résultat, elles ont suggéré que la socialisation des filles leur fournit moins de possibilités de croire en leur capacité d'exercer avec succès les métiers traditionnellement masculins et moins de moyens d'accéder aux informations qui leur permettent de développer leur sentiment de compétence. Si les filles ont moins confiance en elles-mêmes et dans leurs capacités à réussir dans le domaine scientifique et technologique, indépendamment de leur réussite dans ces domaines, elles seront moins motivées à choisir ces carrières. Ainsi, le sentiment de compétence semble être un facteur critique de motivation pour les projets de carrière des individus.
ost-KAMmer et alii, (1985) ont examiné le rôle du sentiment de compétence dans le processus décisionnel de choix de carrière des adolescent(es), et les différences de sexe auprès de 57 filles et 51 garçons scolarisés en $8^{\mathrm{e}}$ et $9^{\mathrm{e}}$ année, à partir d'une enquête par questionnaire. En ce qui concerne les métiers traditionnellement masculins, les garçons et les filles dans cet échantillon ont montré des différences concernant les métiers de dessinateur industriel et d'ingénieur, le sentiment de compétence étant plus grand pour les garçons. En ce qui concerne les métiers traditionnellement féminins, les différences de sexe ont émergé dans la présente étude pour les métiers d'assistant dentaire, de secrétaire, et d'assistant social en faveur des filles. D'après cette enquête, il apparaît que les filles expriment un sentiment de compétence plus élevé pour les métiers «féminins», alors que les garçons ont un sentiment de compétence plus élevé pour les métiers traditionnellement masculins.

En France, les recherches de Blanchard et VRIGNAUD (1994) confirment ces résultats : le sentiment de compétence des garçons est plus élevé pour des professions de technicien en micromécanique, de physicien, d'expert-comptable, d'ingénieur, alors que le sentiment de compétence des filles est plus élevé pour les professions d'éducatrice, de styliste de haute couture, de pédiatre en crèche, d'avocate, de bibliothécaire.

Par ailleurs, il apparaît que les filles ont des aspirations professionnelles aussi élevées que celles des garçons mais n'ont pas l'intention de sacrifier leur famille pour réussir professionnellement (STEVENS, Puchtell, Ryu, Mortimier, 1992). Celles qui expriment des souhaits d'orientation élevés et de réussite de carrière professionnelle envisagent de retarder le mariage et de ne faire que de brèves interruptions après la naissance des enfants. L'étude des stratégies de compromis d'AMSTRONG et Crombie (2000) dans les aspirations professionnelles des adolescent(es) qui ont présenté des différences entre leurs choix idéaliste et réaliste, montre que le changement des aspirations des filles porte sur des métiers traditionnellement féminins, alors que celui des garçons vise les métiers masculins. Les filles ont des aspirations élevées portant majoritairement sur les métiers traditionnellement féminins.

L'importance du sentiment de compétence pour faire face aux obstacles qui s'opposent à la poursuite des études et au choix des carrières scientifiques et techniques chez les filles est mis en évidence dans les recherches de Blaisdell (1993). Dans son étude auprès de 502 lycéens américains, inspirée des théories de Bandura, elle pose l'hypothèse que le sentiment de compétence peut contribuer à expliquer les différences liées au sexe dans le choix de professions scientifiques. Si les filles ont moins confiance en elles-mêmes et dans leurs capacités à réussir dans le domaine scientifique et technologique, 
indépendamment de leur réussite objective dans ces domaines, elles seront moins motivées à choisir des carrières dans l'ingénierie. Les résultats soulignent en effet que, même si les filles avaient un niveau scolaire élevé et une plus grande probabilité de s'inscrire dans des études supérieures scientifiques et technologiques, elles étaient moins nombreuses que les garçons à envisager un métier dans l'ingénierie. Les étudiantes qui ont perçu l'ingénierie comme une carrière enrichissante étaient également moins nombreuses à envisager une telle carrière.

L'étude du développement de la vie professionnelle montre que les garçons et les filles semblent être, tôt dans l'adolescence, également décidés au sujet de leurs carrières. Mais en mûrissant, les filles deviennent plus incertaines au sujet de leurs aspirations de carrière et ont tendance à se conformer aux normes de sexe (Gassin, Kelly, Feldhusen, 1993). Quand il s'agit de l'exploration des carrières, les filles acquièrent moins d'informations sur les différents métiers de façon systématique, et de moins en moins en avançant en âge. Dans leur étude, Flouri et Buchanan (2002) ont enquêté auprès de 2722 adolescent(es) britanniques âgé(e)s de 14 à 18 ans, pour vérifier si l'expérience professionnelle et les modèles professionnels sont associés à la maturité de carrière et aux choix professionnels des adolescent(es). Les résultats indiquent que l'existence chez eux d'un modèle de carrière et d'une première expérience professionnelle est fortement corrélée avec la maturité de carrière plus développée chez les garçons que chez les filles.

La théorie cognitive sociale et le sentiment de compétence développée par Bandura et Betz et Hackett, présume que l'influence des facteurs cognitifs personnels, contextuels et sociaux ont un impact sur la formation de l'intérêt, des buts et de la réalisation de la carrière. La théorie cognitive sociale de la carrière suppose que les variables contextuelles comme l'environnement familial, le degré d'instruction de la mère, les attitudes féministes, etc., exercent une influence sur le sentiment de compétence, qui agit directement sur les intérêts de carrière. L'environnement familial a davantage d'influence sur les ambitions professionnelles des filles que des garçons. Une recherche auprès de filles américaines d'origine mexicaine (FLORES, O'BRIEN, 2002) a montré que le soutien de leurs parents pour la poursuite de leurs études les a aidées à choisir des carrières prestigieuses. Selon ces travaux, l'impact de l'environnement familial sur les projets professionnels des adolescents, et en particulier le modèle de la mère, pourrait influencer les filles et les aider à envisager des carrières scientifiques prestigieuses (O’Brien, FAssinger, 1993).

Le modèle du sentiment de compétence paraît avoir une pertinence particulière pour expliquer le développement de la vie professionnelle des femmes. L'intérêt limité des filles pour des options de l'autre sexe suggère que plus d'attention doit être accordée aux filles qui choisissent d'élargir leurs opinions, notamment dans le domaine des sciences et des technologies.

Le sentiment de compétences des filles pour les carrières scientifiques et techniques est à rechercher dans leurs expériences personnelles, dans leur réussite scolaire dans les matières scientifiques, et dans leur perception positive de la carrière envisagée. Les expériences réalisées par d'autres personnes influencent le sentiment de compétence, et en particulier les expériences des femmes ayant réussi des carrières prestigieuses. Celles-ci peuvent conduire les filles à penser qu'elles seront, elles aussi, capables de réaliser la même tâche après un entraînement approprié ou en suivant la même voie que la personne modèle.

$$
\begin{gathered}
* \\
* \quad *
\end{gathered}
$$

Les recherches françaises ont davantage pour objet d'étudier les représentations des métiers selon le modèle de Gottfredson, en considérant leur statut de masculinité/féminité, alors que les études anglosaxonnes étudient les aspirations professionnelles selon le modèle de Holland qui classe les professions en six catégories. On pourrait se demander si cette classification serait transposable aux représentations des métiers dans le champ français. Le modèle de l'apprentissage social de Bandura qui souligne l'importance du sentiment de compétence dans le choix professionnel se retrouve aussi dans les recherches des deux champs anglophone et francophone.

En tout cas, ces trois modèles ouvrent des pistes de recherche très riches. Cependant les travaux inspirés de ces modèles ne donnent pas d'indications sur les moyens d'augmenter la probabilité des carrières scientifiques chez les adolescentes.

La comparaison entre les deux champs d'études, francophone et anglophone, nous a permis de constater que les recherches françaises ont pour objet d'étudier davantage les représentations des métiers selon leur statut de masculinité/féminité, alors que les études anglo-saxonnes cherchent à examiner les facteurs qui interviennent dans le développement des choix professionnels et dans l'orientation des aspirations professionnelles. Les représentations des jeunes filles et des jeunes gens concernant les métiers et le monde professionnel n'ont pas beaucoup changé dans la période étudiée, entre 1984 et 2004. Les actions mises en place pour lutter contre les stéréotypes sexués concernant les métiers n'ont pas réussi à changer les représentations que les enfants et les adolescent(es) ont du monde du travail, toujours dominées par les stéréotypes de sexe. Il faut reconnaître en même temps, 
que dans la littérature anglo-saxonne de la période récente, les filles commencent à montrer un intérêt pour les métiers traditionnellement masculins. Un nombre croissant de filles envisage et entreprenne d'embrasser des carrières scientifiques et techniques, mais le mouvement est encore timide. Quant aux garçons, leurs modèles restent stéréotypés et ils n'envisagent toujours pas de choisir des métiers dits «féminins». Nous pouvons dire qu'il y a du «mouvement mais pas de changement profond».

Certes, il y a eu, pour les femmes, des transformations indéniables et considérables. En assurant aux femmes l'accès à 1'enseignement secondaire et à l'université, les sociétés occidentales du $\mathrm{XX}^{\mathrm{e}}$ siècle ont permis l'accès des femmes aux savoirs savants et à l'emploi rémunéré, condition d'indépendance économique. Mais il serait illusoire de croire que ce progrès correspond à une suppression pure et simple de la division sociosexuée des savoirs et du travail. Les territoires différenciés n'ont pas été supprimés, ils ont changé de configuration. Il n'y a donc pas eu suppression mais, pour reprendre une expression de Margaret Maruani et Chantal Nicole, «modernisation» de la division sociosexuée des savoirs et du travail (Maruani, Nicole, 1989).

\section{Bibliographie}

\section{Littérature francophone}

Ausseik-Lonka M., Herigult M.-F. (1994), Élude de la structure des représentations professionnelles en fonction du sexe, du positionnement scolaire et de la classe sociale, mémoire DECOP, INETOP.

BAKer M. (1985), Quand je pense à demain... Une étude sur les aspirations des adolescentes, rapport, Conseil consultatif canadien de la situation de la femme, Canada.

BIP (1985), Représentations comparées des filles et des garçons en dernière année d'études secondaires quant à leur avenir social et professionnel. Ingénieur: un métier féminin?

Blanchard S., VRignaud P. (1994), «Intérêts professionnels et sentiment de compétences», Questions d'Orientation, no 4, pp. 31-41.

Bosse N., Guegnard C. (2001), Diversification des choix professionnels. Lumière et ombre des femmes modèles, rapport final pour l'association Orientation au Féminin, délégation régionale des droits des femmes, Fonds social européen.

CAPdebosce M. (1993), Comparaison entre les représentations professionnelles de garçons et de filles de troisième. Évolution de l'effet d'un jeu collectif traitant du monde du travail sur les représentations, mémoire DECOP, INETOP.

CAPEL R. (1995), «Les rêves et projets professionnels des enfants: une étude longitudinale», Orientation et Formation Professionnelle, (article en trois parties) $n^{\circ} 2$, pp. 44-51 (première partie).
CAPel R. (1995), «Les rêves et projets professionnels des enfants: une étude longitudinale. Vers une typologie diachronique «des rêves et projets professionnels remémorés », Orientation et Formation Professionnelle, $\mathrm{n}^{\circ} 3$, pp. 63-68 (seconde partie).

CAPEL R. (1995), «Les rêves et projets professionnels des enfants: une étude longitudinale. De l'enfant au jeune adulte: existe-t-il des invariants de la personnalité basés sur la mesure des intérêts?», Orientation et Formation Professionnelle, $\mathrm{n}^{\mathrm{o}}$ 4, pp. 54-60 (troisième partie).

Chazal S., Guimond S. (2003), «La théorie de la dominance sociale et les choix d'orientation scolaire et des rôles sociaux des filles et des garçons», Orientation Scolaire et Professionnelle, no 4, vol. 32, pp. 595-616.

Costechareyre C., Solazzi R. (2002), «Enquête sur les représentations et images de métiers de jeunes scolarisés en collège », L'Indécis, no 47, pp. 5-42.

Dumora B. (2002), «L'imaginaire professionnel des jeunes adolescents», Carriérologie revue francophone internationale, vol. 8, no 3 .

Feuilladieu S. (2001), Projets de lycéens. Orientation et projets en classe de seconde générale et technologique, Paris, L'Harmattan.

FONTANINI Ch. (2001), «Quels sont les freins à l'orientation des filles vers les classes préparatoires scientifiques MP, $\mathrm{PC}$ et $\mathrm{PCI} »$, Questions d'Orientation, no 2, pp. 15-32.

Fromage B., Guion G., Vioux E. (2001), «Images adolescentes dans l'orientation professionnelle», Revue de psycho-éducation et d'orientation, vol. 30, no 2, pp. 349363. 
Gazet B., Brosseau D. (1985), Étude de l'impact de différents attributs des métiers sur les préférences professionnelles en fonction du niveau scolaire, de sexe et de la catégorie socioprofessionnelle des parents, mémoire DECOP, INETOP.

Guegnard C. (2001), Diversification des choix professionnels des filles. Filles et lycée technique, des résistances? Rapport final pour l'association Orientation au Féminin, délégation régionale des droits des femmes, Fonds social européen.

Guegnard C., Bertrand E., Louis C. (2002), Diversification des choix professionnels des filles. Le carrefour des carrières au féminin, l'idée d'un autre métier, rapport final pour l'association Féminin Technique (FETE), délégation régionale des droits des femmes, Fonds social européen.

Guegnard C. (2002), «Représentations professionnelles des filles et des garçons au collège. Les effets d'une pièce de théâtre interactive», Orientation Scolaire et Professionnelle, no 4, vol. 31, pp. 601-622.

Guglielmi D., Fraccarolli F., Pombeni L.-M. (2004), «Les intérêts professionnels selon le modèle hexagonal de Holland. Structure et différences de genre», Orientation Scolaire et Professionnelle, vol. 33, n 3, pp. 409-427.

Guichard J., Bidot H. (1989), «Filières scolaires et représentations professionnelles des lycéens», Revue Internationale de Psychologie Sociale, no 4, pp. 485-509.

Guichard J. (1992), «Échec scolaire et représentations de soi et des professions », Orientation Scolaire et Professionnelle, $\mathrm{n}^{\circ}$ 2, pp. 149-162.

Guichard J. (1993), L'École et les représentations d'avenir des adolescents, Paris, PUF.

Guichard J. et alii (1994), «Diversité et similarité des représentations professionnelles d'adolescents scolarisés dans des formations différentes », Orientation Scolaire et Professionnelle, $n^{\circ}$ 4, vol. 23, pp. 409-437.

Guichard J., Devos P., Bernard H., Chevalier G., Devaux M., Faure A., Jellab M., Vanesse V. (1994), «Habitus culturels des adolescents et schèmes représentatifs des professions », Orientation Scolaire et Professionnelle, no 4, vol. 23, pp. 439-464.

Huteau M., Marro C. (1986), Les connotations du mot «travail»chez les lycéens, Laboratoire de psychologie différentielle service de recherche de l'INETOP.

Huteau M, Vouillot F. (1988), «Représentations et préférences professionnelles», Bulletin de psychologie, tome $42, n^{\circ} 388$, pp. 144-153.

Kessili-Faddel S. (2000), Les représentations professionnelles d'adolescents scolarisés dans les structures différents, mémoire de DECOP, INETOP.

LEMERCIER-KUHN D. (1991), «Les motivations professionnelles d'élèves de $3^{\mathrm{e}}$. Aspects généraux », première partie, Bulletin de l'ACOF, vol. 54, no 4, pp. 19-33.

LEMERCIER-KUHN D. (1992), «Les motivations profes- sionnelles d'élèves de $3^{\mathrm{e}}$. Aspects différentiels et interculturels », deuxième partie, Bulletin de l'ACOF, no 337 , pp. 56-70.

Leo-Dupon E., Gendre F., Chaghaghi F., Muller B. (1984), «Les déterminants des aspirations professionnelles des adolescents (15-16 ans)», Orientation et Formation Professionnelle, no 5, pp. 279-285.

LOPES A. (1997), Les projets d'orientation des élèves de $3 e$ des Mureaux, mémoire de maîtrise, sciences de l'éducation, Paris X-Nanterre.

Mariotti F. (2001), «Place et statut des mathématiques selon le sexe dans la structure des représentations sociales de la science et des métiers scientifiques chez les collégiens et des lycéens », Les cahiers internationaux de psychologie, $\mathrm{n}^{\circ} 49$, pp. 78-96.

Mariotti F. (2002), «La sexuation de l'implication à s'engager dans un métier scientifique au lycée", Recherches féministes, volume XV, $\mathrm{n}^{\mathrm{0}} 1$, pp. 47-65.

Marro C. (1989), «Les choix scolaires et professionnels d'élèves de seconde envisageant une première scientifique », Orientation Scolaire et Professionnelle, $\mathrm{n}^{\circ} 3$, pp. b251-262.

Marro C., Vouillot F. (1991), «Représentation de soi, représentation du scientifique-type et choix d'une orientation scientifique chez des filles et des garçons de seconde», Orientation Scolaire et Professionnelle, $\mathrm{n}^{\circ} 3$, vol. 20, pp. 303-323.

TERLON C. (1990) «Attitudes des adolescents à l'égard de la technologie», Revue française de pédagogie, $\mathrm{n}^{\circ} 90$, pp. 51-59.

ThiBault A. (1995), Les filles et les métiers non traditionnels : du mouvement mais pas de changement, coordination à la condition féminine, gouvernement du Québec, ministère de l'Éducation.

Vouillot F., Blanchard S., Marro C., Steinbruckner M.-L. (2004), «La division sexuée de l'orientation et $\mathrm{du}$ travail: une question théorique et une question de pratiques », Psychologie du travail et des organisations, 10, pp. 277-291.

WACH M. (1992), «Projets et représentations des études et des professions des élèves de $3^{\mathrm{e}}$ et de terminale en $1992 »$, Orientation Scolaire et Professionnelle, n 3 , vol. 21, pp. 297-339.

WACH M., (1993), «Les représentations du travail. Une enquête auprès de jeunes en lycée professionnel», Éducation permanente, $\mathrm{n}^{\mathrm{o}} 117$, pp. 113-120.

Zitouni A. (2003), Projet USA. Égalité des chances, mémoire, haute école de gestion, Vaud, Suisse.

\section{Littérature anglo-saxonne}

Armstrong I.P., Crombie G. (2000), “Compromises in Adolescents Occupational Aspirations and Expectations from Grades 8 to 10", Journal of Vocational Behavior, no 56, pp. 82-98. 
Blaisdell S. (1993), "Predictors of women's entry into engineering: Why academic preparation is not sufficient", Journal of Vocational Behavior, no 45, pp. 79-122.

Brooks L., Holahan W., Galligan M. (1985), "The Effects of a Nontraditional Role-Modeling Intervention on Sex Typing of Occupational Preferences and Career Salience in Adolescent Females", Journal of Vocational Behavior, $\mathrm{n}^{\circ}$ 26, pp. 264-276.

Constance L. Hotlinger E., Fleming S. (1993), "Project CHOICE: The Emerging Roles and Careers of Gifted Women", Roper Review, vol. 15, no 3, pp. 156-160.

Dunnell P. A., BAKken L. (1991), "Gifted high school students'attitudes toward careers and sex roles", Røepr Review, vol. 13, pp. 198-202.

Flouri E., Buchanan A. (2002), "The role of work-related skills and career role models in adolescent career maturity - Special Section : Adolescent Career Development", Career Development Quarterly, September.

Flores Y.L., O'Brien M.K. (2002), “The Career Development of Mexican American Adolescent Women : A Test of Social Cognitive Career Theory", Journal of Counseling Psychology, vol. 49, n 1, pp. 14-27.

Gassin E.A., Kelly K.R., Feldhusen J.F. (1993), "Sex differences in the career development of gifted youth", The School Counselor, vol. 41, pp. 90-95.

Grotevant D.H., Cooper R.C., Kramer K. (1986), "Exploration as a Predictor of Congruence in Adolescents Career Choices", Journal of Vocational Behavior, n 29 , pp. 201-215.

LAPAN T.R., Jingeleski J. (1992), “Circumscribing Vocational Aspirations in Junior High School”, Journal of Counseling Psychology, vol. 39, n 1, pp. 81-90.

LAuver J.Ph., Jones M.R. (1991), "Factors Associated With Perceived Career Options in American Indian, White, and Hispanic Rural High School Students", Journal or Counseling Psychology, vol. 38, n 2, pp. 159-166.

LAwrie L., Brown R. (1992), "Sex stereotypes, school subject preferences, and career aspirations as a function of single/mixed-sex schooling and presence/absence of an opposite sex sibling", British Journal of Educational Psychology, vol. 62, pp. 132-138.

Martin-Rainey L., Borders D. (1997), "Influential Factors in Career Orientation and Career Aspiration of Early Adolescent Girls", Journal of Counseling Psychology, vol. 44, n 2, pp. 160-172.

Mau W.C., Bikos L.H. (2000), "Educational and Vocational Aspirations of Minority and Female Students : A Longitudinal Study", Journal of Counseling and Development, vol. 78, pp. 186-195.

MAU W.C. (2003), "Factors that influence persistence in science and engineering career aspirations", Career Development Quarterly, mars.

Mcmahon M., Patton W. (1997), “Gender differences in children's and adolescents'perceptions of influences on their career development", School Counselor, 44, pp. 368-376.

Mcnulty B.W., Borgen A.W. (1988), "Career Expectations and Aspirations of Adolescents", Journal of Vocational Behavior, no 33, pp. 217-224.

O’Brien K.M., FASSINGer R.E. (1993), “A causal model of the career orientation and career choice of adolescent women", Journal of Counseling Psychology, 40, pp. 456469.

Patton W., Creed A.P. (2001), "Developmental Issues in Career Maturity and Career Decision Status", Career Development Quarterly, juin.

Perron M.K., Sedlacek E.W., Alexander M.C. (2001), "Gender and Ethnic Differences in Career Goal Attainment", Career Development Quarterly, December.

Post-Kammer P., Smith P.L. (1985), "Sex differences in career self-efficacy, consideration, and interests of eighth and ninth grades", Journal of Counseling Psychology, vol. $32, \mathrm{n}^{\mathrm{o}} 4$, pp. 551-559.

Sandberg D.E., Ehrhardt A.A., Ince S.E., MeyerBAHLBURG H.F.L. (1991), "Gender differences in children's and adolescents'career aspirations: A follow-up study", Journal of Adolescent Research, 6, pp. 371-386.

Stevens C.J.,Puchtell L.A., RyuS., Mortimer J.T. (1992), "Adolescent work and boys'and girls'orientation to the future", The Sociological Quarterly, 33, pp. 153-169.

Stockard J., McGee J. (1990), “Children's Occupational Preferences: The Influence of Sex and Perceptions of Occupational Characteristics", Journal of Vocational Behavior, no 36 , pp. 287-303.

Strange C.C., Rea S.J. (1983), "Career Choice Considerations and Sex Role Self-Concept of Male and Female Undergraduates in Nontraditional Majors", Journal of Vocational Behavior, no 23, pp. 219-226.

Taveira Do Ceu M., Silva C., Rodriguez L.M., Maia J. (1998), "Individual characteristics and career exploration in adolescence", British Journal of Guidance and Counseling, vol. 26, n 1, pp. 89-102.

TURNER S., LAPIN T.R.. (2002), “Career self-efficacy and perceptions of parent support in adolescent career development", Career Development Quarterly, September.

Turner L.S., Steward C.J., Lapin T.R. (2004), "Family factors associated with sixth-grade adolescents'math and science career interests", Career Development Quarterly, September.

Watson C.M., Quatman T., Edler E. (2002), "Career aspirations of adolescent girls: effects of achievement level, grade, and single-sex school environment", Sex Roles: A journal of research, May.

Wulff B.M., Steitz A.J. (1997), "Curricular track, career choice, and androgyny among adolescent, females", Adolescence, Winter. 


\section{Bibliographie générale}

Bandura A. (1977), "Self-efficacy: Toward a unifying theory of behavioral change", Psychological Review, 84 (2), pp. 191-215.

Bandura A. (1982), "Self-efficacy mechanism in human agency", American Psychologist, 7, pp. pp. 122-147.

Betz N.E., Наскетt G. (1981), "The relationship of career-related self-efficacy expectations to perceived career options in college women end man", Journal of Counseling Psychology, 28 (5), pp. 399-410.

Combes D., Daune-Richard A.-M., Devreux A.M. (1991), «Mais à quoi sert une épistémologie des rapports sociaux de sexe?», in M.-C. HuRTIG, M. KaIL, $\mathrm{H}$. Rouch, Sexe et genre. De la hiérarchie entre les sexes, Paris, CNRS.

Couppie T., Epiphane D. (2001), «Que sont les filles et les garçons devenus?», Marseille, Céreq Bref, nº 178.

EHESIA (1995), La place des femmes. Les enjeux de l'identité et de l'égalité au regard des sciences sociales, Paris, La Découverte.

GotTFRedSON L. (1981), "Circumscription and compromise: A developmental theory of occupational aspirations", Journal of Counseling Psychology, 28, 6, pp. 545-597.

Holland J.L. (1966), The psychology of vocationnal choice, Waltham, MA: Blasidell.

Hurtig M-C., Kail M., Rouch H. (sous la direction de), (1991), Sexe et genre: de la hiérarchie entre les sexes, Paris, CNRS (réédition 2002).

Kergoat, D. (2000), «Division sexuelle du travail et rapports sociaux de sexe», in H. Hirata, F. Laborie,
F. Le Doaré, D. SÉnotier, (Eds), Dictionnaire critique du féminisme, Paris, PUF.

Le Maner-Idrissi G. (1997), L'identité sexuée, Paris, Dunod.

Le Maner-Idrissi G., Lévêque A., Massa J. (2002), «Manifestations précoces de l'identité sexuée», Orientation Scolaire et Professionnelle, 31 (4), pp. 507522.

MARro, C. (1998), «La tolérance à la transgression des rôles de sexe chez l'adolescent(e)», Pratiques Psychologiques, 3, pp. 39-50.

Maruani M., Nicole Ch., (1989), Au labeur des dames. Métiers masculins, emplois féminins, Paris, Syros, Alternatives.

Mathieu N.C. (2000), «Sexe et genre», in H. Hirata, F. Laborie, F. Le Doaré, D. SÉnotier (Eds), Dictionnaire critique du féminisme, Paris, PUF.

Pichevin M.-F. (1995), «De la discrimination sociale entre les sexes aux automatismes psychologiques: serions-nous tous sexistes?», in collectif, La place des femmes. Les enjeux de l'identité et de l'égalité au regard des sciences sociales, Ephesia, Paris, La Découverte.

Vouillot F., Marro C. (2002), numéro spécial: «Construction et affirmation de l'identité chez les filles et les garçons, les femmes et les hommes de notre société», Orientation Scolaire et Professionnelle, 31 (4).

Vouillot F., Blanchard S., Marro C., steinbrukner M.-L. (2004), «La division sexuée de l'orientation et du travail: une question de politiques», Psychologie du travail et des organisations, $\mathrm{n}^{0}$ spécial (10), pp. 277-291. 